\title{
Challenging Axillary Lymph-Nodes on F-18-FDG, F- 18-Choline and Ga-68-DOTATOC PET/CT in Cancer Patients throughout Covid-19 Vaccination Era
}

\section{Giovanni Storto ( $\sim$ giosto24@hotmail.com )}

Istituto di Ricovero e Cura a Carattere Scientifico (IRCCS), Referral Cancer Center of Basilicata (CROB)

\section{Alessia Giordano}

Istituto di Ricovero e Cura a Carattere Scientifico (IRCCS), Referral Cancer Center of Basilicata (CROB)

\section{Rosj Gallicchio}

Istituto di Ricovero e Cura a Carattere Scientifico (IRCCS), Referral Cancer Center of Basilicata (CROB)

\section{Milella Mariarita}

Istituto di Ricovero e Cura a Carattere Scientifico (IRCCS), Referral Cancer Center of Basilicata (CROB)

Teresa Pellegrino

Istituto di Ricovero e Cura a Carattere Scientifico (IRCCS), Referral Cancer Center of Basilicata (CROB)

Antonio Nappi

Istituto di Ricovero e Cura a Carattere Scientifico (IRCCS), Referral Cancer Center of Basilicata (CROB)

Anna Nardelli

Istituto di Ricovero e Cura a Carattere Scientifico (IRCCS), Referral Cancer Center of Basilicata (CROB)

Giovanni Calice

Istituto di Ricovero e Cura a Carattere Scientifico (IRCCS), Referral Cancer Center of Basilicata (CROB)

\section{Research Article}

Keywords: Covid-19, PET/CT, cancer, lympho-adenopathy

Posted Date: March 7th, 2022

DOI: https://doi.org/10.21203/rs.3.rs-1393065/v1

License: (c) (i) This work is licensed under a Creative Commons Attribution 4.0 International License.

Read Full License 


\section{Abstract}

Objectives: unexpected detection of axillary lymphadenopathy $(A x L)$ in cancer patients (Pts) represents a real concern during COVID-19 vaccination era. Benign reactions may take place after vaccine inoculation, confounding image interpretation in patients undergoing F-18-FDG, F-18-Choline and Ga-68-DOTATOC PET/CT. It may simulate loco-regional metastases/disease. To assess PET/CT findings after COVID-19 vaccination in cancer patients and the consequent impact on their management.

Methods: we evaluated 333 patients undergoing PET/CT (257 F-18-FDG, 54 F-18-Choline and 23 Ga-68 DOTATOC) scans after first vaccination with mRNA vaccine (Pfizer-BioNTech). Uptake index (SUVmax) of suspected $A x L$ was defined significant when the ratio $>1.5$ as compared to the contralateral inoculation site. Besides, co-registered CT (Co-CT) features of target lymph-nodes were evaluated. Nodes with aggregate positivity were further investigated.

Results: overall, the prevalence of apparently positive lymph-nodes on PET scans was $17.1 \%$ during vaccination era. $107 \mathrm{Pts}$ had undergone PET/CT before COVID-19 pandemic and only 3 shown reactive lymph-nodes with a prevalence of $2.8 \%$ ( $p<0.001$ as compared to vaccination era). $84.2 \%$ exhibited benign characteristics on Co-CT images and only 9 Pts needed additional appraisal.

Conclusions: the correct interpretation of images is crucial to avoid unnecessary management changes and to rule out invasive procedures in cancer Pts undergoing diagnostics. An accurate anamnestic interview and the precise assessment of nodes' Co-CT characteristics when performing PET/CT may help to address the diagnostic hypothesis.

\section{Key Points}

1. The correct interpretation of PET/CT images avoid unnecessary management changes in cancer patients undergoing SARS-CoV-2 vaccination

2. The prevalence of apparently positive lymph-nodes on PET scans was higher during vaccination era as compared to that of patients who had undergone PET/CT before COVID-19 pandemic.

3. A significant number of patients exhibited benign characteristics on Co-registered-CT images and only few needed additional appraisals.

\section{Introduction}

The coronavirus disease 2019 (Covid-19) pandemic has caused millions of deaths with great commitment by world health services [1]. The scientific endeavor has led to the development of vaccines useful to reduce infections, transmissibility, and mortality [2]. The Pfizer/BioNTech vaccine was the first SARS-CoV-2 (Covid-19) vaccine to be approved by Regulatory Agencies [3-4], followed by the Oxford/AstraZeneca and Moderna vaccines [5]. The administration of vaccines has been a priority for health workers and the most fragile patients such as elderly and immunosuppressed [6]. 
Mild systemic symptoms like fatigue, myalgia, and headache along with locoregional adverse reactions, such as shoulder pain and erythema have been reported after COVID-19 vaccination, as for other types of vaccines [7-8]. However, recent data have suggested a higher incidence of local reactive lymphadenopathy in the axillary and neck regions at the side of injection as compared to other vaccines [9]. With the increasing use of vaccines, the presence of lymphadenopathies has become a diagnostic dilemma for cancer patients. In this setting the differential diagnosis concerning adenopathy is mandatory to exclude metastatic or recurrent cancers [10]. Accordingly, the United States Society of Breast Imaging, the Canadian Society of Breast Imaging, the Canadian Association of Radiologists, and a multidisciplinary team (MDT) of experts from three leading cancer centers in USA [11] have released recommendations about the management of lymphadenopathy due to Covid-19 vaccination. Cases of ipsilateral adenopathy in the axillary and supraclavicular regions following deltoid Covid- 19 vaccinations are quickly emerging in the international literature and are being increasingly referred to healthcare services for advice and management [12].

The PET/CT (Positron Emission Tomography - Computed Tomography) holds a widely validated and recognized role in cancer diagnostics [13-15]. On the other hand, the most common radiotracers used in $\mathrm{PET} / \mathrm{CT}$ imaging are not cancer specific at all [13]. Among the others the radio-labeled glucose analogue F-18-2-fluoro-2-deoxyglucose (F-18-FDG) is the most employed tracer in PET imaging. It accumulates both in cancer and non-cancer cells having high rate of glycolysis, such as activated leukocytes in inflammatory conditions [16]. As a result, the clinical use of (F-18-FDG) PET/CT for assessing inflammatory and infectious diseases is increasing worldwide [17]. Moreover, the tracer F-18-Choline is used for the appraisal of prostate cancer. It is a radiolabeled precursor of phosphatidylcholine, an essential phospholipid of cell membranes. Both in cancer cells and reactive lymph-nodes the enhanced choline uptake is explained by the increased mitogenic activity, which requires more membrane precursors [18]. Finally, radiolabeled somatostatin analogues, such as Ga-68-DOTATOC, -DOTANOC and DOTATATE are utilized to assess neuroendocrine tumors expressing somatostatin receptors.

Nevertheless, lymphatic tissues including reactive lymph-nodes might accumulate this radioligand [1920].

We aimed to study PET/CT findings after COVID-19 vaccination in cancer patients and the consequent impact on their management.

\section{Materials And Methods}

\subsection{Patients}

We assessed 333 oncological patients (overall; 143 women; 65 13 years) who underwent F-18-FDG (132 women; $63 \pm 13$ years), or F18-Choline (all men; 74 8 years) or Ga-68-DOTATOC (11 women; 60 \pm 13 years) PET/CT scans from March to May 2021 for staging, re-staging or follow-up assessment. Aggregated individual data are presented in table 1. 
Table 1

Patients' individual data 


\begin{tabular}{|c|c|}
\hline Characteristics & Values \\
\hline Total number of patients & 333 \\
\hline Age at enrollment, year, median (range) & $65(21-97)$ \\
\hline Sex M:F & $190: 143$ \\
\hline \multicolumn{2}{|l|}{ Cancer diagnosis, pts (\%) } \\
\hline Prostate & $56(16.8)$ \\
\hline Gastro-enteric & $44(13.2)$ \\
\hline Breast & $42(12.6)$ \\
\hline Lymphomas and hematological diseases & $39(11.7)$ \\
\hline Lung & $38(11.4)$ \\
\hline Gynecologic & $28(8.4)$ \\
\hline Head and Neck & $25(7.5)$ \\
\hline GEP/non-GEP NET & $23(6.9)$ \\
\hline Melanomas & $9(2.7)$ \\
\hline Urothelial & $9(2.7)$ \\
\hline Testis & $5(1.5)$ \\
\hline Thyroid & $4(1.2)$ \\
\hline Others & $11(3.3)$ \\
\hline \multicolumn{2}{|l|}{ PET/CT diagnostic inquiry, pts (\%) } \\
\hline Staging & $93(27.9)$ \\
\hline Re-staging & $156(46.9)$ \\
\hline Follow-up & $84(25.2)$ \\
\hline \multicolumn{2}{|c|}{ PET suspected lymphadenopathy (female/male) } \\
\hline$F-18-F D G$ & $31 / 16$ \\
\hline$F-18-F C H$ & $0 / 8$ \\
\hline Ga-68-DOTATATE & $0 / 2$ \\
\hline
\end{tabular}

GEP, Gastroenteropancreatic; NET, Neuroendocrine Tumors; Others, Thymus cancer, Sarcomas, Unknown origin; PET/CT, Positron Emission Tomography/Computed Tomography; F-18-FDG, F-18-2-fluoro-2- 
deoxyglucose; F-18-FCH, F-18-fluoro-choline; Ga-68-DOTATATE, Gallium-68-DOTA-0-Tyr3-Octreotate

From the beginning of COVID-19 vaccination era, all patients referred either for F-18-FDG, F-18-Choline or Ga-68-DOTATOC PET/CT scans were invited to report about the COVID-19 vaccination type they received, as well as timing and anatomical site of inoculation. To make our observations as homogeneous as possible, only patients vaccinated with the first dose of Pfizer-BioNTech, presenting age at entry of 18 years or older and negative pregnancy test were evaluated. Patients had undergone comprehensive reevaluation including clinical and oncological data. Insufficient information about the kind of vaccine and/or the anatomical region of injection constituted a reason for exclusion. Patients whose lymph-node sites were previously treated by recent external radiotherapy before PET/CT imaging were excluded from this study to avoid confounding items.

We also collected data from PET/CT scans in the same patients before COVID 19 pandemic, if any, as reference.

Procedures included in the study were submitted to our institutional review board for approval. All patients who underwent the study signed an informed consent form in accordance with the Declaration of Helsinki.

\subsection{Imaging technique}

The patients were well-hydrated before receiving F-18-FDG (370-555 MBq), F-18-Choline (230-300 MBq) or Ga-68-DOTATOC (250-300 MBq) via a peripheral venous catheter on the contralateral site of vaccination. Sixty minutes after the tracer injection, PET and modulated low dose CT were carried out with a commercial PET/CT scanner (GE Discovery VCT scanner) that combined a PET scanner and a Light Speed VCT sixty-four row MDCT system. MDCT (pitchx 1.5; 120 mAs; 120 kVp) was performed without contrast medium. The PET scanning was subsequently performed, acquiring 3 min per bed position and six to eight bed per patient depending on patient height encompassing the whole body. The raw CT data were reconstructed into transverse images with a 3.75-mm section thickness. Sagittal and coronal CT images were generated by reconstruction of the transverse data. Raw PET data were reconstructed with and without attenuation correction into transverse, sagittal, and coronal images. Attenuation correction was based on CT attenuation coefficients, which were determined by iterative reconstruction. Patients were fasted and blood glucose levels were determined only before F-18 FDG administration (cut-off value of less than $8.3 \mathrm{mmol} / \mathrm{L} ; 150 \mathrm{mg} / \mathrm{dL}$ ).

\subsection{Imaging evaluation}

All hybrid images were reviewed by using PET/CT fusion software (Volumetrix for PET-CT and AW volume share 4.5, GE Healhcare). The PET studies were evaluated both visually and semi-quantitatively by SUVmax by two nuclear medicine physicians with 20 yrs of expertise. Then, the SUVmax body weight 
corrected values were determined by using the vendor-provided software. According to the method previously used by Thomassen et al. [21], only vaccine-draining lymph-nodes (target lesion) that had shown a SUVmax ratio $\geq 1.5$ when compared to the contralateral site, were circumspectly studied on CT.

Bilaterality and multi-centricity were assessed, if any. All target lesions were visually assessed for shape and hilus characteristics and measured at CT in one maximum diameter at least, in short axis images by using the above-mentioned vendor-provided software. A target lesion was defined as reactive lymph-node if two of the three abovementioned features indicated a benign nature (oval in shape; clearly evident hilus; $<10 \mathrm{~mm}$ diameter) with soft-tissue/mediastinal window settings, along with metabolic findings. Patients with lesions mismatching with normal anatomy or physiology and suspected of disease recurrence/relapse were referred for ultrasound and biopsy, if any. Study algorithm is reported in figure 1.

\subsection{Follow-up assessment}

Patients for whom a short follow-up PET/CT imaging was scheduled according to clinical needs, were reevaluated for both metabolic and morphological findings.

\section{Statistical Analysis}

Statistical analysis was performed using MedCalc Software version 20.014. Numeric variables and frequencies of categorical variables were calculated. Fisher's exact test was used to evaluate the proportions of incidental findings observed before and after vaccinations. A p value $<0.05$ was considered statistically significant.

\section{Results}

Two hundred fifty-six patients who underwent F-18- FDG PET/CT scan, 54 who received F-18-Choline and 23 referred for Ga-68-DOTATOC were eligible, respectively.

Overall, the prevalence of apparently positive axillary lymph-nodes on PET scans was $17.1 \%$ during vaccination era whereas it was $18.3 \%$ for patients who received F-18-FDG, $14.8 \%$ for F-18-Choline and $8.7 \%$ Ga-68-DOTATOC, respectively.

Among the 333 patients 107 had undergone PET/CT before COVID-19 pandemic and only 3 shown reactive lymph-nodes with a prevalence of $2.8 \%$ ( $p<0.001$ as compared to vaccination era).

The range of SUVmax in positive lymph-nodes, irrespective of the tracer used, was 1.8 to 4.7 , with a mean SUVmax of $2.8 \pm 1.1$. Data of the metabolic results according to the tracer employed are shown in table 2. The average time elapsed from vaccination was of $16 \pm 7$ days for F-18- FDG, $22 \pm 5$ days for the F-18Choline and $26 \pm 7$ days Ga-68-DOTATOC, respectively.

\section{Table 2}


SUVmax and timing from vaccination in cancer patients with hyper-metabolic lymph-nodes according to radiotracers

\begin{tabular}{|llllll|}
\hline $\begin{array}{l}\text { PET/CT } \\
\text { tracers }\end{array}$ & $\begin{array}{l}\text { Total scans } \\
\text { post } \\
\text { vaccination } \\
\text { March-May } \\
2021\end{array}$ & $\begin{array}{l}\text { Suspected Axillary } \\
\text { lymphadenopathy } \\
\#(\%)\end{array}$ & $\begin{array}{l}\text { SUVmax } \\
\text { Mean } \\
(\text { SD })\end{array}$ & $\begin{array}{l}\text { SUVmax } \\
\text { Range }\end{array}$ & $\begin{array}{l}\text { Average time } \\
\text { elapsed from } \\
\text { vaccination } \\
\text { (days) }\end{array}$ \\
\hline$F^{18}$ FDG & 257 & $47(18.3)$ & $\begin{array}{l}2.95 \pm \\
1.2\end{array}$ & $2.0-4.7$ & $16 \pm 7$ \\
\hline$F^{18}$ Choline & 54 & $8(14.8)$ & $\begin{array}{l}2.36 \pm \\
0.9\end{array}$ & $1.8-2.9$ & $22 \pm 5$ \\
\hline Ga ${ }^{68}$ DOTATOC & 23 & 2 (8.7) & $\begin{array}{l}2.27 \pm \\
0.7\end{array}$ & $1.9-2.6$ & $26 \pm 7$ \\
\hline $\begin{array}{l}\text { SUVmax, Standard Uptake Value; F-18-FDG, F-18-2-fluoro-2-deoxyglucose; F-18-FCH, F-18-fluoro- } \\
\text { choline; Ga-68-DOTATATE, Gallium-68-DOTA-0-Tyr3-Octreotate }\end{array}$ & & \\
\hline
\end{tabular}

Fifty-seven patients had an apparently positive lymph-node on PET/CT scan (hypermetabolic/receptor overexpression; Fig. 2), none bilateral, two patients shown multiple ipsilateral nodes.

Among them 48 (84.2\%) showed benign characteristics on co-recorded CT images and were considered disease-free avoiding further investigations. Nine patients (15.8\%) presented suspected CT features (round shape, max diameter $>10 \mathrm{~mm}$ and/or not evident hilus), 7 patients underwent ultrasound appraisal that excluded adenopathy disease, two had a further mdc CT; only for one of them a metastatic disease was evoked.

Twenty-six of 57 (45.6\%) patients who apparently presented positive lymph-node on PET scan throughout the vaccination era have had a PET/CT also before COVID-19 pandemic; none showed suspected axillary nodes at that time.

No muscular and subcutaneous uptake on the deltoid region were observed. The shortest time range from vaccination was 6 days, when normally the non-specific deltoid's muscular uptake has already disappeared.

Also, twenty-eight patients of 57 (49.1\%) with previous target lesion received a negative short-term PET/CT follow-up within three months. Of note, 23/47 (49\%) patients who underwent F-18-FDG PET/CT and exhibited suspected axillary nodes suffered from cancers such as breast, melanoma and lymphoma, where the presence or the absence of lymphatic spread would have really changed their management. None had disease confirmed. 


\section{Discussion}

To our knowledge this is the first study assessing the PET/CT findings with different tracers after COVID19 vaccination in cancer patients and the consequent impact on their management.

The prevalence of locoregional PET positive lymph-nodes after SARS-CoV-2 vaccine inoculation was significantly higher as compared to pre-COVID-19 era.

After recently vaccine administration reactive lymphadenopathy in the ipsilateral axillary and/or supraclavicular area is most likely to occur. Unfortunately, it represents a serious challenge in cancer patients who undergo F-18-FDG, since it might mimic disease onset, relapse, or recurrence [22-25] whereas for F-18-Choline and Ga-68 DOTATOC PET/CT scans it may constitute only an unusual finding. Our patients were referred for staging, re-staging or follow-up to PET/CT examinations with different radioligands according to their diagnostic inquiry. Incidentally, the rate of apparently positive lymphnodes was significant in this setting. Although our findings may appear not astonishing, they deserve caution, particularly for cancer patients as recently reported by some other authors [23-25]. The correct interpretation of this secondary effect of vaccine is of primary importance to avoid unnecessary changes in patients' management and to rule out undue biopsies as well as to differentiate them from malignancies, recurrence and/or metastases.

We focused on the importance of a correct anamnestic interview concerning recent vaccination in oncological patients undergoing diagnostics and particularly on the precise appraisal of co-recorded CT features when they go through PET/CT scans. In this study patients were invited to report about vaccine inoculation from the beginning of COVID-19 vaccination era. In addition, suspected lymph-nodes on the PET scan were reviewed on co-registered CT for malignancy [25]. According to daily practice, nodes with aggregate positivity were further investigated by US or mdc CT, at least [26]. This approach avoided undue further investigations for most of patients and unnecessary invasive procedures [27] for those supposed positive. In fact, $84 \%$ of patients suspected positive on PET scan resulted to have benign features on co-recorded CT images. In only one case imaging confirmed malignancy.

The prevalence of the abovementioned incidental findings was significant for all the tracers investigated according to their different frequency in use (see Ga-68 peptides). From a physio-pathological point of view, F-18 FDG, as glucose analogue, distributes in both cancer and inflammatory glucose-utilizing cells $[16-17,28]$ and, with different pathways, inflammatory cells may concentrate the F-18-Choline and Ga68-DOTATOC [18-20].

Recently, some authors [23-25] have reported similar results but, in smaller cohorts, sometimes in form of case report, using one tracer and after adenovirus-based vaccine which is known to elicit inflammatory response [29]. Our study was conducted collecting data from 333 consecutive patients, irrespective of the tracer used, who underwent mRNA SARS-CoV-2 vaccination and reporting on findings both in pre-COVID19 period and thereafter in a subset of patients. Almost $50 \%$ of patients had PET/CT scan negative in this two moments confirming the incidental nature of the observations during vaccination age. 
Moreover, we renew the importance of the correct interpretation of these incidental findings during metabolic imaging in cancer patients to prevent unjustified misapprehension and the temporal enlargement of diagnostic path, as well as unnecessary changes in therapy and management.

When PET positive, the uptake index (SUVmax) was significant in all the three radiotracers satisfying the method of Thomassen [21] and justifying the need for further caution.

Concerning the nodes' metabolic positivity duration few information are still available. It seems that reactive processes to vaccination may last up to 70 days [30-31]. Indeed, the longer the time elapsed the lower the tracers' uptake intensity once scans performed newly. Accordingly, in our patients the difference in SUVmax values between the radiotracers could be explained by the time elapsed from vaccination and the different pharmacodynamic characteristics as showed in table 2.

Finally, we suggest careful interpretation of PET/CT data in cancer patients who recently undergo SARSCoV-2 vaccine (and others vaccine as well) [32-36] considering the vaccine site of injection and addressing for subsequent imaging appraisal for the ambiguous lymph-nodes to confirm or reject the diagnostic hypothesis. In doubtful cases, the biopsy remains the last useful tool.

\section{Perspectives}

We aim to collect more data, especially from patients vaccinated with different vaccines, to evaluate any differences in terms of uptake's intensity and duration as well as from patients after the second and/or the booster dose.

\section{Abbreviations}

AxL: axillary lymphadenopathy

Pts: patients

F-18-FDG: fluoride-18-2-fluoro-2-deoxyglucose

Ga-68 DOTATOC: gallium-68 DOTA-D-Phe1-Tyr3-octreotide

PET/CT: positron emission tomography/computed tomography

SUVmax: maximum standardized uptake

Co-CT: co-registered computed tomography

-DOTANOC:(1,4,7,10-tetraazacyclododecane-N,N',N",N"'-tetraacetic acid)-1-Nal3-octreotide

-DOTATATE: DOTA-0-Tyr3-Octreotate 


\section{Declarations}

The authors declare that no funds, grants, or other support were received during the preparation of this manuscript.

The authors have no relevant financial or non-financial interests to disclose.

The datasets generated during and/or analyzed during the current study are available from the corresponding author on reasonable request.

\section{References}

1. Viscusi, W K. Pricing the global health risks of the COVID-19 pandemic. Journal of Risk and Uncertainty. 61(2):101-128 (2020).

2. Jabłońska, K., Aballéa, S., Toumi, M. The real-life impact of vaccination on COVID-19 mortality in Europe and Israel. Public Health.198:230-237 (2021). doi: 10.1016/j.puhe.2021.07.037.

3. Tanne, JH. Covid-19: FDA approves Pfizer-BioNTech vaccine in record time. BMJ. 374:n2096 (2021). doi: 10.1136/bmj.n2096.

4. lacobucci, G. Covid-19: FDA set to grant full approval to Pfizer vaccine without public discussion of data. BMJ. 374:n2086 (2021). doi:10.1136/bmj.n2086 pmid:34417195

5. Su, S., Du, L., Jiang, S. Learning from the past: development of safe and effective COVID-19 vaccines. Nat Rev Microbio/19(3):211-219 (2021). doi: 10.1038/s41579-020-00462-y

6. Fanciullino, R., Ciccolini, J., Milano, G. COVID-19 vaccine race: watch your step for cancer patients. BJC. 124(5),860-861(2021).

7. Kau,r S.P., Gupta, V. COVID-19 Vaccine: A comprehensive status report. Virus res.288:198114 (2020). doi: 10.1016/j.virusres.2020.198114.

8. Rodríguez-Alfonso, B., Ruiz Solís, S., Silva-Hernández, L., Pintos Pascual, I., Aguado Ibáñez, S., Salas Antón, C. ${ }^{18}$ F-FDG-PET/CT in SARS-CoV-2 infection and its sequelae. Rev Esp Med Nucl Imagen Mol (Engl Ed). 40(5):299-309 (2021). doi: 0.1016/j.remnie.2021.07.005.

9. Ahn, R.W., Mootz, A.R., Brewington, C.C., Abbara, S. Axillary lymphadenopathy after mRNA COVID-19 vaccination. Radiology: Cardiothoracic Imaging. 3(1) (2021). e210008.

10. McIntosh, L.J., Bankier, A.A., Vijayaraghavan, G.R., Licho, R., Rosen, M.P. COVID-19 vaccinationrelated uptake on FDG PET/CT: an emerging dilemma and suggestions for management. AJR Am J Roentgenol. 217(4):975-983 (2021). doi: 10.2214/AJR.21.25728.

11. Seely, J.M., Barry, M.H. The Canadian Society of Breast Imaging/Canadian Association of Radiologists' Recommendations for the management of axillary adenopathy in patients with recent COVID-19 vaccination. Can Assoc Radiol J.72(4):601-602 (2021). doi: 0.1177/0846537121998949.

12. Lehman, C.D., D’Alessandro, H.A., Mendoza, D.P., Succi, M.D., Kambadakone, A., Lamb, L.R. Unilateral lymphadenopathy after COVID-19 vaccination: a practical management plan for radiologists across 
specialties. J Am Coll Radiol.18(6):843-852. (2021).

13. Bar-Shalom, R., Valdivia, A.Y., Blaufox, M.D. (2000, July). PET imaging in oncology. Semin Nucl Med. 30(3):150-185 (2020).

14. Storto, G., Nicolai, E., Salvatore, M. [(18) F] FDG-PET-CT for early monitoring of tumor response: when and why. Q J Nucl Med Mol Imaging. 53:167-180 (2009).

15. Storto, G., et al. Assessment of metabolic response to radioimmunotherapy with 90Y-ibritumomab tiuxetan in patients with relapsed or refractory B-cell non-Hodgkin lymphoma. Radiology.254(1):245252 (2010). doi: 10.1148/radiol.09090603.

16. Treglia, G. Diagnostic performance of 18F-FDG PET/CT in infectious and inflammatory diseases according to published meta-analyses. Contrast Media Mol Imaging. 2019:3018349 (2019). doi: $10.1155 / 2019 / 3018349$

17. Casali, M., et al. State of the art of 18F-FDG PET/CT application in inflammation and infection: a guide for image acquisition and interpretation. Clin Transl Imaging.10:1-41 (2021). doi: 10.1007/s40336-021-00445-w.

18. Oprea-Lager, D.E., et al. Dual-phase PET-CT to differentiate [18F] Fluoromethylcholine uptake in reactive and malignant lymph nodes in patients with prostate cancer. PLOS One.7:48430 (2012).

19. Treglia, G., Castaldi, P., Rindi, G., Giordano, A., Rufini, V. Diagnostic performance of gallium-68 somatostatin receptor PET and PET/CT in patients with thoracic and gastroenteropancreatic neuroendocrine tumours: a meta-analysis. Endocrine42:80-87 (2012).

20. Weiler-Sagie, M., Elizabeth, E.H. [68 Ga] Ga-DOTA-TATE uptake due to COVID-19 vaccination. Eur J Nucl Med Mol Imaging. 22:1-2 (2021).

21. Thomassen, A., Lerberg Nielsen, A., Gerke, O., Johansen, A., Petersen, H. Duration of 18F FDG avidity in lymph nodes after pandemic H1N1v and seasonal influenza vaccination. Eur J Nucl Med Mol Imaging. 38: 894-898 (2011).

22. Smith, M.V., Yang, M. Reactive Axillary Lymphadenopathy to COVID-19 Vaccination on 18 F-FDG PET/CT. J Nucl Med Technol. 49(3):286-287 (2021). doi: 10.2967/jnmt.121.262008.

23. Nawwar, A.A., Searle, J., Hopkins, R., Lyburn, I.D. False-Positive Axillary Lymph Nodes on FDG PET/CT Resulting From COVID-19 Immunization. Clin Nucl Med. 46(12):1004-1005 (2021). doi: 10.1097/RLU.0000000000003657.

24. Schroeder, D.G., et al. Frequency and Characteristics of Nodal and Deltoid FDG and (11)C-Choline Uptake on PET Performed After COVID-19 Vaccination. AJR Am J Roentgenol. 17(5):1206-1216 (2021). doi: 10.2214/AJR.21.25928. Epub 2021 May 19.

25. Eifer, M., Eshet, Y. Imaging of COVID-19 Vaccination at FDG PET/CT. Radiology.299(2):E248 (2021). doi: $10.1148 /$ radiol.2020210030.

26. Cocco, G., et al. Lymphadenopathy after the Anti-COVID-19 Vaccine: Multiparametric Ultrasound Findings. Biology (Basel). 10(7):652 (2021). doi: 10.3390/biology10070652. 
27. Tan, N.J.H., Tay, K.X.J., Wong, S.B.J., Nga, M.E. COVID-19 post-vaccination lymphadenopathy: Report of cytological findings from fine needle aspiration biopsy. Diagn Cytopathol. 49(12):E467E470 (2021). doi: 10.1002/dc.24863.

28. Albano, D., et al; Prevalence of interstitial pneumonia suggestive of COVID-19 at 18F-FDG PET/CT in oncological asymptomatic patients in a high prevalence country during pandemic period: a national multi-centric retrospective study. On the behalf of Italian Association of Nuclear Medicine (AIMN). Eur J Nucl Med Mol Imaging. 48(9):2871-2882 (2021). doi: 10.1007/s00259-021-05219-0.

29. Shin, M., Hyun, C.Y., Choi, Y.H., Choi, J..Y, Lee, K.H., Cho, Y.S. COVID-19 Vaccination-Associated Lymphadenopathy on FDG PET/CT: Distinctive Features in Adenovirus-Vectored Vaccine. Clin NuCl Med. 46(10):814-819 (2021). doi: 10.1097/RLU.0000000000003800.

30. Eshet, Y., Tau, N., Alhoubani, Y., Kanana, N., Domachevsky, L., Eifer, M. Prevalence of Increased FDG PET/CT Axillary Lymph Node Uptake Beyond 6 Weeks after mRNA COVID-19 Vaccination. Radiology.300(3):E345-E347 (2021). doi: 10.1148/radiol.2021210886.

31. Panagiotidis, E., Exarhos, D., Housianakou, I., Bournazos, A., Datseris, I. FDG uptake in axillary lymph nodes after vaccination against pandemic (H1N1). Eur Radiol. 20:1251-1253 (2010). doi: 10.1007/s00330-010-1719-5.

32. Su, N., Wiefels, C., Klein, R., Zeng, W., Abbaspour, F. Intensity of hypermetabolic axillary lymph nodes in oncologic patients in relation to timeline following COVID-19 vaccination. J Med Imaging Radiat Sci.20:S1939-8654(22)00002-9 (2022). doi: 10.1016/j.jmir.2022.01.004

33. Bshesh, K., et al. Lymphadenopathy post-COVID-19 vaccination with increased FDG uptake may be falsely attributed to oncological disorders: A systematic review. J Med Virol. 20. (2022). doi: 10.1002/jmv.27599.

34. Weissman, J.P., Bartel, T.. COVID-19 messenger ribonucleic acid vaccination and abnormal radiopharmaceutical uptake in the axilla visualized on 68Ga-DOTATATE positron-emission tomography/computed tomography. World J Nucl Med.20(4):392-394 (2021). doi: 10.4103/wjnm.wjnm_54_21.

35. Tsumura, Y., Asakura, K., Takahash,i I., Akaihata, M., Takahashi, Y., Ishida, Y. New mimic of relapse or regional lymph node metastasis in a cancer survivor: a case of mRNA COVID-19 vaccine-induced lymphadenitis with high FDG uptake. Immunol Med.16:1-3 (2021). doi: 10.1080/25785826.2021.1999786.

36. Arens, A.I.J., Hebeda, K.M., Hutchings, M., Plattel, W.J., Stevens, W.B.C. Axillary lymph nodes on PET in Hodgkin lymphoma after COVID-19 vaccination. EJHaem.2(4):885-886 (2021). doi: 10.1002/jha2.297.

\section{Figures}




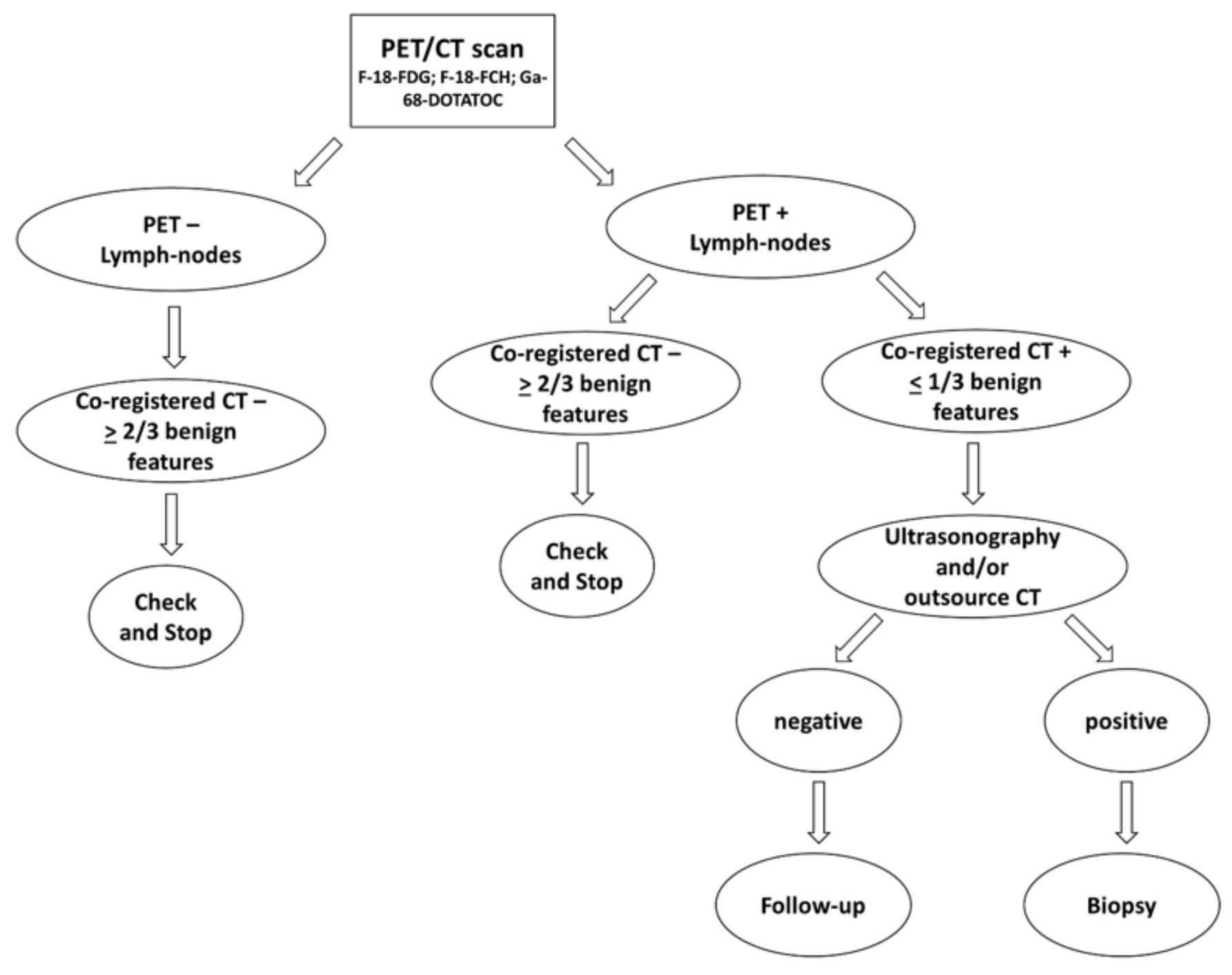

Figure 1

study algorithm. PET/CT: positron emission tomography/computed tomography; F-18-FDG: F-18-2fluoro-2-deoxyglucose; F-18-FCH: F-18-Choline; Ga-68-DOTATOC: Ga-68 somatostatin analogue; Coregistered CT: CT acquired during hybrid PET/CT; CT benign features: maximum diameter $<10 \mathrm{~mm}$, evident hilus, oval shape; outsource CT: additional CT scan. 

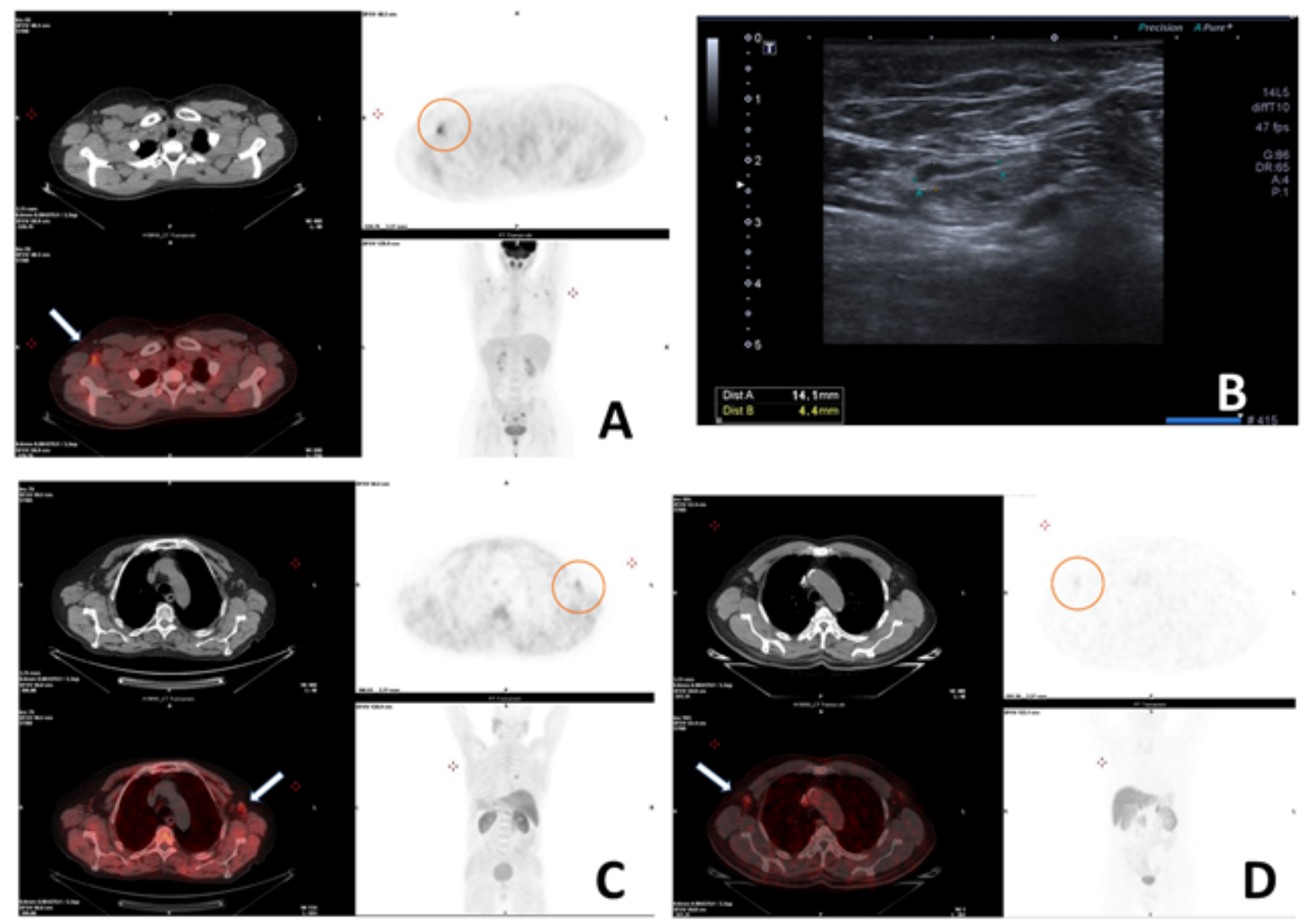

Figure 2

PET/CT scans $(A ; C ; D)$ in cancer patients showing doubtful axillary lymphadenopathy after mRNA SARS.CoV-2 vaccination. A: F-18-FDG scan in 35 yrs-old Hodgkin lymphoma woman with suspected lymph-nodes in right axilla on PET and benign features on both co-registered CT and subsequent ultrasound (B, diameters are shown). C: F-18-Choline in 83 yrs-old man complying with castrate sensitive metastatic prostate cancer presenting left axillar lymph-node uptake and benign characteristics on coregistered CT. D: Ga-68-DOTATOC in 72 yrs-old man exhibiting gastroenteropancreatic (GEP-NET) cancer, with PET positive right axillary lymph-node and negative co-registered CT. 